\title{
Chromosomal organization of repetitive DNAs in Hordeum bogdanii and $H$. brevisubulatum (Poaceae)
}

\author{
Quanwen Dou', Ruijuan Liu', Feng Yu' \\ I Key Laboratory of Adaptation and Evolution of Plateau Biota, Northwest Plateau Institute of Biology, Chinese \\ Academy of Sciences, Xining 810001, China \\ Corresponding author: Quanwen Dou (douqw@nwipb.cas.cn)
}

$\frac{\text { Academic editor: V. Shneyer | Received 24 June } 2016 \mid \text { Accepted } 12 \text { August } 2016 \mid \text { Published } 7 \text { October } 2016}{\text { http://zoobank.org/E306475B-BE5D-4E19-A06D-70FEB1A371C6 }}$

Citation: Dou Q, Liu R, Yu F (2016) Chromosomal organization of repetitive DNAs in Hordeum bogdanii and $H$. brevisubulatum (Poaceae). Comparative Cytogenetics 10(4): 465-481. doi: 10.3897/CompCytogen.v10i4.9666

\begin{abstract}
Molecular karyotypes of $H$. bogdanii Wilensky, $1918(2 \mathrm{n}=14)$, and $H$. brevisubulatum Link, 1844 ssp. brevisubulatum $(2 \mathrm{n}=28)$, were characterized by physical mapping of several repetitive sequences. A total of 18 repeats, including all possible di- or trinucleotide SSR (simple sequence repeat) motifs and satel-

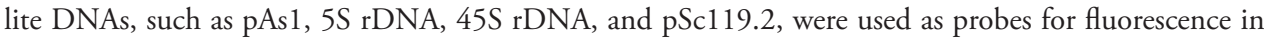
situ hybridization on root-tip metaphase chromosomes. Except for the SSR motifs AG, AT and GC, all the repeats we examined produced detectable hybridization signals on chromosomes of both species. A detailed molecular karyotype of the I genome of $H$. bogdanii is described for the first time, and each repetitive sequence is physically mapped. A high degree of chromosome variation, including aneuploidy and structural changes, was observed in $H$. brevisubulatum. Although the distribution of repeats in the chromosomes of $H$. brevisubulatum is different from that of $H$. bogdanii, similar patterns between the two species imply that the autopolyploid origin of H. brevisubulatum is from a Hordeum species with an I genome. A comparison of the I genome and the other Hordeum genomes, $\mathrm{H}, \mathrm{Xa}$ and $\mathrm{Xu}$, shows that colocalization of motifs AAC, ACT and CAT and colocalization of motifs AAG and AGG are characteristic of the I genome. In addition, we discuss the evolutionary significance of repeats in the genome during genome differentiation.
\end{abstract}

\section{Keywords}

Hordeum bogdanii, Hordeum brevisubulatum, autopolyploid, repetitive sequence, FISH

Copyright Quanwen Dou et al. This is an open access article distributed under the terms of the Creative Commons Attribution License (CC BY 4.0), which permits unrestricted use, distribution, and reproduction in any medium, provided the original author and source are credited. 


\section{Introduction}

Species in Triticeae have large genomes, $75 \%$ of which consists of repetitive sequences (Flavell et al. 1974, 1977). Many repetitive sequences, such as microsatellite and satellite DNA, can generate fluorescence in situ hybridization (FISH) patterns on individual chromosomes that are specific to a single species (Tsujimoto et al. 1997, Cuadrado et al. 2008). The FISH patterns generated by these repetitive probes always produce a stable and unique karyotype for a given species (Badaeva et al. 1996, 2002). The FISH patterns from a few repeats correspond to the heterochromatin regions in chromosomes (Cuadrado et al. 1995, Pedersen and Langridge 1997). Thus, the genomic organization of a given species can be characterized using FISH-based cytological analysis. Phylogenies can even be derived from repeat-based comparative FISH karyotyping (Cuadrado and Jouve 2002, Jiang and Gill 2006, Heslop-Harrison and Schwarzacher 2011).

The genus Hordeum Linnaeus, 1753 in the tribe Triticeae is divided into 32 species and is distributed in southern South America, South Africa, and the northern hemisphere (Bothmer et al. 1995). Cytotypes in this genus exist at three ploidy levels (diploid, tetraploid and hexaploid) with a basic chromosome number of $x=7$ (Bothmer et al. 1995). Hybrid analysis and C-band analysis (Bothmer et al. 1986, 1995, Linde-Laursen et al. 1992) have revealed four genome groups in Hordeum that are designated as H (Hordeum bulbosum Linnaeus, 1756; Hordeum vulgare Linnaeus, 1753), $\mathrm{Xa}$ (Hordeum marinum Hudson, 1778), Xu (Hordeum murinum Linnaeus, 1753), and I (all the remaining species) (Blattner 2009). The $\mathrm{H}$ genome chromosomes of $\mathrm{H}$. vulgare were first characterized using FISH probes of all possible classes of trinucleotide SSRs (simple sequence repeat). This provided detailed information on the sequence content of barley chromatin and saturated the physical map of all the barley chromosomes (Cuadrado and Jouve 2007a). Later, cytogenetic diversity between $H$. vulgare and $H$. bulbosum, was revealed using probes of SSRs, $5 \mathrm{~S}$ rDNA, $45 \mathrm{~S}$ rDNA, and 120 bp repeats from Secale cereale Linnaeus, 1753 (Carmona et al. 2013a). Karyotypes of the species of the $H$. marinum complex were determined using several tandem repeats. The results revealed the genome structure of different $H$. marinum taxa and demonstrated the allopolyploid origin of tetraploid forms of $H$. gussoneanum Parlatore, 1845 (Carmona et al. 2013b). In addition, the genomic constitution of $H$. murinum was characterized using multiple repetitive sequences. The results identified all the individual chromosomes within the $H$. murinum complex, elucidated its genomic structure and phylogeny, and explained the appearance of different cytotypes (Cuadrado et al. 2013). Thus far, detailed information on the chromosome organization of multiple repetitive sequences is available for the $\mathrm{H}, \mathrm{Xa}$, and $\mathrm{Xu}$ genomes. However, this information is still lacking for the I genome.

Hordeum bogdanii Wilensky, 1918, and H. brevisubulatum, should include the I genome based on the description of Blattner (2009). H. bogdanii is a rather common Asiatic species with a distribution that ranges from western Iran to eastern China and a diploid form of $2 \mathrm{n}=2 \mathrm{x}=14$ (Yang et al. 1987). Conversely, the $H$. brevisubulatum complex is distributed from Western Turkey to eastern China and consists of diploids, 
tetraploids, and hexaploids (Bothmer 1979, Linde-Laursen et al. 1980). Polyploids of $H$. brevisubulatum are thought to be autoploid based on morphological (Bothmer 1979, Dewey 1979) and hybrid meiotic analysis (Landström et al. 1984).

In this paper, the molecular karyotype of the I genome is described in detail based on physical mapping of all possible dinucleotide and trinucleotide SSRs along with $5 \mathrm{~S}$ rDNA, $45 \mathrm{~S}$ rDNA, pSc119.2, and pAs1 repeats on mitotic chromosomes in $H$. bogdanii and $H$. brevisubulatum. The results provide more information on the genomic differentiation in Hordeum at the chromosomal level and will also help elucidate the functional and evolutionary implications of different repetitive sequences as genomes differentiate during speciation.

\section{Material and methods}

\section{Plant material}

Hordeum bogdanii was collected in Germu, Qinghai, China. Hordeum brevisubulatum ssp. brevisubulatum was collected in Tongde, Qinghai, China. More than 50 or more than 100 individuals of both species were collected in the field. Samples used for cytogenetic investigation were randomly selected from different individuals. Approximately 20 individuals were used for chromosome preparation. Only the investigated samples that displayed clear FISH patterns were present in this study.

\section{Slide preparation}

Seeds of $H$. bogdanii and $H$. brevisubulatum were germinated on moist filter paper in petri dishes at room temperature. Root tips with a length of approximately $1-2 \mathrm{~cm}$ were excised, pretreated in $\mathrm{N}_{2} \mathrm{O}$ gas for $2 \mathrm{~h}$ as described by Kato (1999), and fixed in 3:1 (v/v) 100\% ethanol:glacial acetic acid. Each root tip was squashed in a drop of $45 \%$ acetic acid.

\section{DNA probes and labelling}

Synthetic dinucleotide SSRs $(\mathrm{AT})_{15},(\mathrm{AG})_{15},(\mathrm{AC})_{15}$, and $(\mathrm{GC})_{15}$ and trinucleotide SSRs $(\mathrm{AAG})_{10},(\mathrm{AAC})_{10},(\mathrm{AAT})_{10},(\mathrm{ACG})_{10},(\mathrm{ACT})_{10},(\mathrm{AGG})_{10},(\mathrm{CAC})_{10},(\mathrm{CAG})_{10},(\mathrm{CAT})_{10}$ and $(\mathrm{GGC})_{10}$ were end-labelled with fluorescein amidate (FAM, green, Sangon Biotech Co., Ltd., Shanghai, China). For the repetitive sequences pAs1 (Rayburn and Gill 1986), pSc119.2 (Bedbrook et al. 1980), and 45S rDNA, oligonucleotide probes described by Danilova et al. (2012) and Tang et al. (2014) were used. The oligonucleotides pSc119.2 and 45S rDNA were end-labelled with FAM (green), and pAs1 was end-labelled with TAMRA (red). The $5 \mathrm{~S}$ rDNA was amplified by polymerase chain 
reaction (PCR) using genomic DNA of $H$. bogdanii as described by Fukui et al. (1994) and was labelled with fluorescein-12-dUTP (green) using a random primer labelling method. Genomic DNA of $H$. bogdanii was labelled with tetramethy1-rhodamine5-dUTP using the random primer method, as described by Dou et al. (2009).

\section{FISH and microphotometry}

FISH experiments were conducted using the method of Dou et al. (Dou et al. 2009) with minor modifications. Samples on prepared slides were denatured in $0.2 \mathrm{M} \mathrm{NaOH}$ in 70\% ethanol at room temperature for $10 \mathrm{~min}$, rinsed in $70 \%$ cold ethanol (stored at minus $20^{\circ} \mathrm{C}$ ) for approximately 30 minutes, and air dried. The hybridization mixture, $10 \mu \mathrm{l}$ per slide, was prepared by adding $50 \%$ de-ionized formamide, $50 \%$ dextran sulphate, $2 \times \operatorname{SSC}(0.3 \mathrm{M} \mathrm{NaCl}, 0.03 \mathrm{M} \mathrm{Na} 3$-citrate $), 1 \mu \mathrm{g} / \mu \mathrm{l}$ denatured salmon sperm DNA and $10 \mathrm{ng}$ of probe. The hybridization mixture with oligonucleotide probes was placed directly onto the denatured slide preparation. The hybridization mixture with the $5 \mathrm{~S}$ rDNA probe was denatured in boiling water for 5 minutes. Hybridization was conducted overnight at $37^{\circ} \mathrm{C}$ in a moistened chamber. Chromosomes were counterstained with 4',6-diamidino-2-phenylindole (DAPI). Images were captured with a cooled CCD camera (Photometrics CoolSNAP) using a fluorescence microscope (Leica) and were processed with the Meta Imaging System (Universal Imaging Corporation). Finally, images were adjusted using Adobe Photoshop 6.0 for contrast and background optimization.

\section{Results}

\section{Chromosomal organization of repeats in $\mathrm{H}$. bogdanii}

A stable chromosome number of $2 \mathrm{n}=14$ was detected in all tested samples of $H$. bogdanii. The repetitive sequence pAs1 produced multiple sites that were subtelomeric, intercalary, or pericentromeric on all chromosomes. The hybridization pattern of pAs1 was informative enough to distinguish each chromosome of $H$. bogdanii. Thus, chromosome localization of other repeats was conducted using pAs1 as a landmark (Fig. 1). The chromosomal distribution of each sequence was described in detail (Fig. 2).

The four possible dinucleotide SSR probes $(\mathrm{AG})_{15},(\mathrm{AC})_{15},(\mathrm{AT})_{15}$, and $(\mathrm{GC})_{15}$ were used to characterize the chromosomes of $H$. bogdanii. Only $(\mathrm{AC})_{15}$ produced detectable hybridization signals, which appeared in subtelomeric regions on all chromosomes and in pericentromeric regions on a few chromosomes. The signals were strongest in subtelomeric regions of chromosomes B, C and D (Fig. 2).

All 10 trinucleotide SSRs produced detectable hybridizations (Fig. 2). The probe $(\mathrm{AAC})_{10}$ revealed many intense and rich pericentromeric hybridization signals on all seven pairs of chromosomes, intercalary signals on chromosomes B and F, and subtelo- 

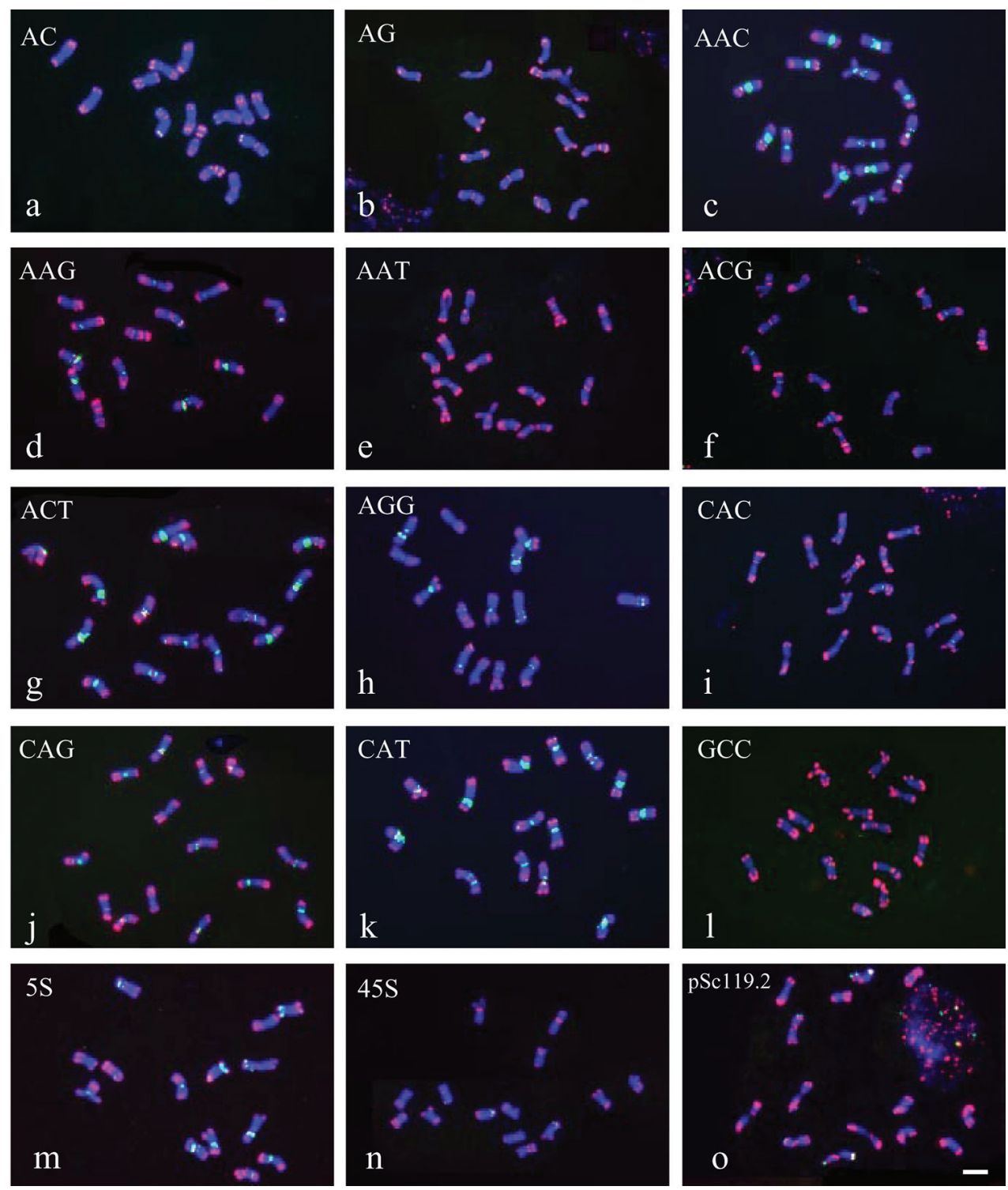

Figure I. FISH patterns of mitotic metaphase chromosomes of $H$. bogdanii detected by pAs1 (red) combined with the several other repeats (green): a (AC) ${ }_{15} \mathbf{b}(\mathrm{AG})_{15} \mathbf{c}(\mathrm{AAC})_{10} \mathbf{d}(\mathrm{AAG})_{10}$ e (AAT) ${ }_{10}$ $\left.\mathbf{f}(\mathrm{ACG})_{10} \mathbf{g}(\mathrm{ACT})_{10} \mathbf{h}(\mathrm{AGG})_{10} \mathbf{i}(\mathrm{CAC})_{10} \mathbf{j}(\mathrm{CAG})_{10} \mathbf{k} \mathrm{CAT}\right)_{10} \mathbf{I}(\mathrm{GCC})_{10} \mathbf{m} 5 \mathrm{~S}$ rDNA $\mathbf{n}$ 45 SrDNA o pSc119.2. Bar $=10 \mu \mathrm{m}$.

meric signals on chromosomes D and E. The FISH patterns of (ACT) ${ }_{10}$ and (CAT) ${ }_{10}$ appeared to be identical to those of $(\mathrm{AAC})_{10}$. An identical FISH pattern was shared by $(\mathrm{AAG})_{10}$ and $(\mathrm{AGG})_{10}$, which produced intense pericentromeric hybridizations, intercalary hybridizations, or both in six pairs of chromosomes. The (CAC) ${ }_{10}$ probe produced faint signals in four chromosome pairs and strong signals in three pairs in peri- 


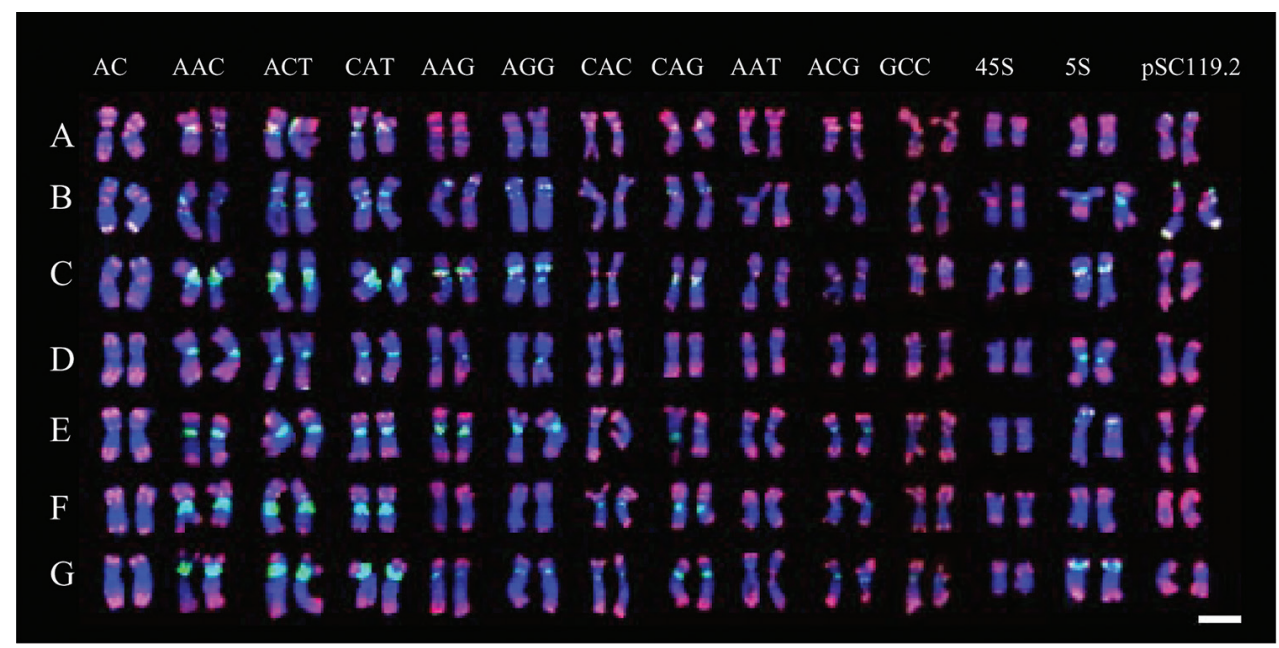

Figure 2. Molecular karyotypes of $H$. bogdanii probed by pAs1 (red) combined with the other several repeats (green). Seven pairs of chromosomes are designated from $\mathbf{A - G}$ for distinguishing them from the numerals of the 7 homologue groups used in barly. Bar $=10 \mu \mathrm{m}$.

centromeric regions, intercalary regions, or both. The distribution of the (CAG) ${ }_{10}$ signal overlapped with that of the $(\mathrm{CAC})_{10}$ signal, but the $(\mathrm{CAG})_{10}$ signal had a stronger hybridization intensity. (AAT) ${ }_{10}$ produced faint signals in three pairs of chromosomes in pericentromeric, intercalary, and subtelomeric regions. Hybridization of (ACG) ${ }_{10}$ was detected in six pairs of chromosomes and was localized primarily in centromeric regions. The distribution of (GCC) ${ }_{10}$ was more dispersed; however, more intense hybridizations were still observed in centromeric regions on three pairs of chromosomes.

Three $45 \mathrm{~S}$ rDNA sites were detected in two pairs of chromosomes. One carried a distinct site on the subtelomeric region of the short arm; the other harboured two faint hybridization sites in the ends of both arms. $5 \mathrm{~S}$ rDNA was distributed in all chromosomes except for one. Nine distinct $5 \mathrm{~S}$ rDNA sites were exclusively determined and were localized on centromeric, pericentromeric, intercalary, or subtelomeric regions. Three pairs of chromosomes carried only one $5 \mathrm{~S}$ rDNA site, and another three harboured two $5 \mathrm{~S}$ rDNA sites. Two $45 \mathrm{~S}$ rDNA and three $5 \mathrm{~S}$ rDNA sites were reported in an accession of $H$. bogdanii (Taketa et al. 2001). The fact that many more $45 \mathrm{~S}$ rDNA and $5 \mathrm{~S}$ rDNA sites were revealed in this study suggests the presence of intra-specific polymorphisms. Four distinct hybridization sites were probed by $\mathrm{pSc} 119.2$, and these were localized on subtelomeric regions of three pairs of chromosomes.

\section{Chromosomal organization of repeats in $\mathrm{H}$. brevisubulatum}

A chromosome number of $2 \mathrm{n}=28$ was detected in nearly all tested individuals of $H$. brevisubulatum. However, chromosome numbers of $2 n=26$ and $2 n=27$ (Fig. 3a, d) 

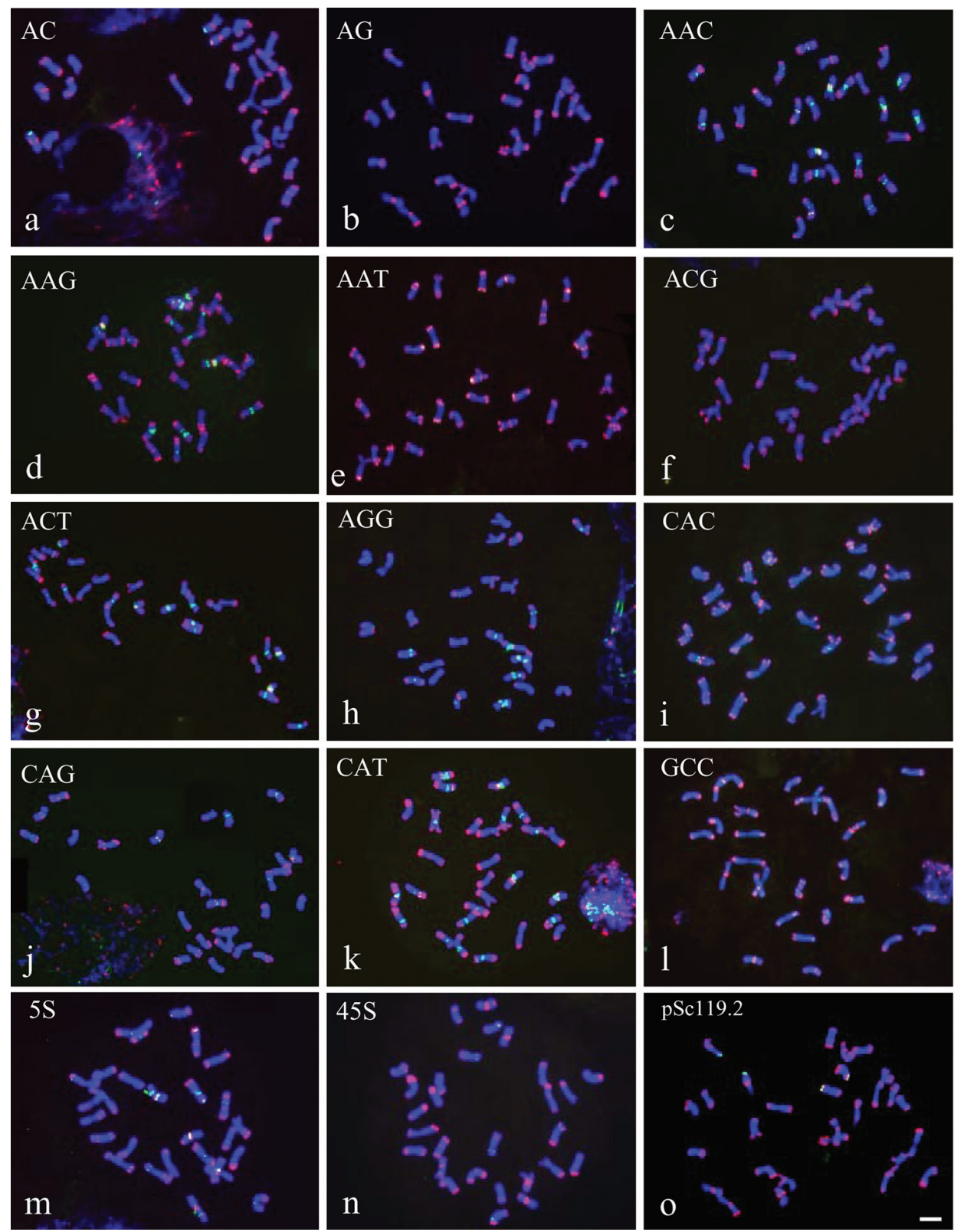

Figure 3. FISH patterns of mitotic metaphase chromosomes of $H$. brevisubulatum detected by pAs1 (red) combined with several other repeats (green): a (AC) ${ }_{15} \mathbf{b}(\mathrm{AG})_{15} \mathbf{c}(\mathrm{AAC})_{10} \mathbf{d}(\mathrm{AAG})_{10}$ e (AAT) ${ }_{10}$ $\mathbf{f}(\mathrm{ACG})_{10} \mathbf{g}(\mathrm{ACT})_{10} \mathbf{h}(\mathrm{AGG})_{10} \mathbf{i}(\mathrm{CAC})_{10} \mathbf{j}(\mathrm{CAG})_{10} \mathbf{k}(\mathrm{CAT})_{10} \mathbf{I}$ (GCC) ${ }_{10} \mathbf{m} 5 \mathrm{~S}$ rDNA $\mathbf{n} 45 \mathrm{SrDNA}$ o pSc119.2. Bar $=10 \mu \mathrm{m}$.

were also observed in a few cases. Moreover, a monotelosomic chromosome was clearly identified in one case (Fig. 3k). Karyotyping was tentatively conducted using pAs 1 combined with other repeats. Highly variable karyotypes were revealed in H. brevisubulatum 


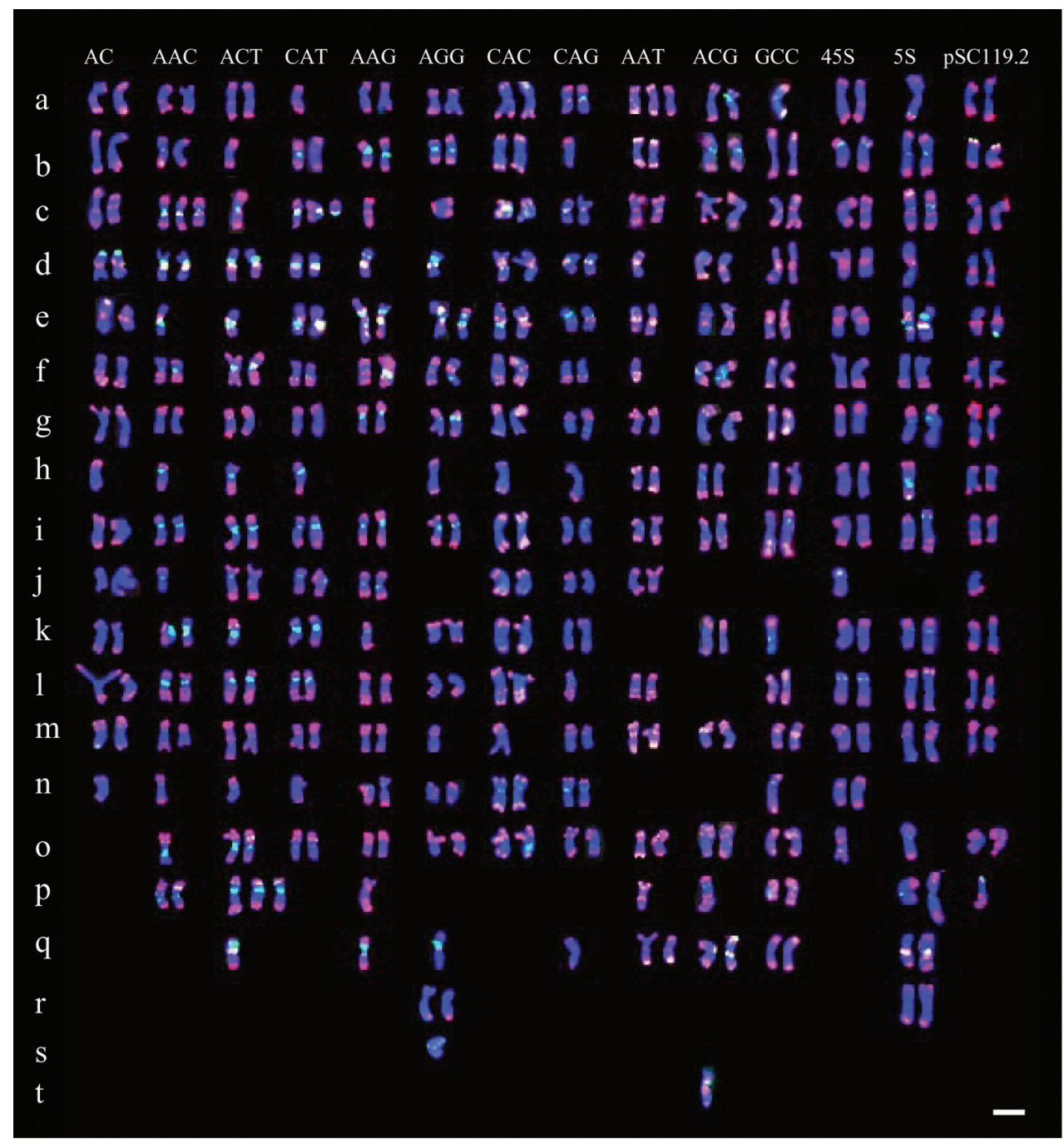

Figure 4. Molecular karyotypes of H. brevisubulatum probed by pAs1 (red) combined with several other repeats (green). Chromosome types are designated by lowercase roman letters from a to $t$ to distinguish them from the symbols designating $H$. bogdanii. Bar $=10 \mu \mathrm{m}$.

(Fig. 4). Variations in chromosome number, such as monosomy, nullisomy, and trisomy, were clearly shown to have occurred. Chromosome structural aberrations, such as deletions, translocations, or inversions, are likely to be present, although these aberrations were not evident from the FISH results alone. Thus, approximately 20 chromosomal variants designated from a to $t$ were roughly identified in the individuals that were investigated (Fig. 4).

All possible di- and trinucleotide SSR probes except for $(\mathrm{AG})_{15},(\mathrm{AT})_{15}$ and $(\mathrm{GC})_{15}$ produced hybridizations on chromosomes of $H$. brevisubulatum. The $(\mathrm{AC})_{15}$ probe 


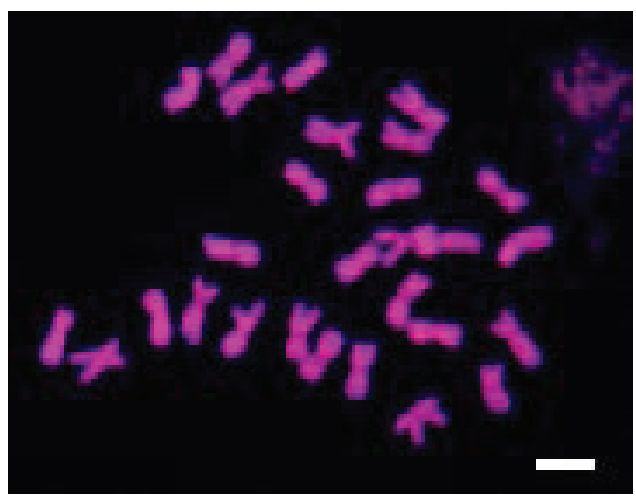

Figure 5. GISH patterns of mitotic metaphase chromosomes of $H$. brevisubulatum probed by the genomic DNA of $H$. bogdanii.

was primarily detected in subtelomeric regions. Nearly half of the total chromosomes carried detectable signals, and signals of seven or eight chromosomes appeared more distinct. The chromosomal distribution of $(\mathrm{AAC})_{10},(\mathrm{ACT})_{10}$, and $(\mathrm{CAT})_{10}$ was similar to that in $H$. bogdanii. The $(\mathrm{AAC})_{10},(\mathrm{ACT})_{10}$, and $(\mathrm{CAT})_{10}$ repeats were still colocalized and were detected on 22-26 chromosomes. (AAG) ${ }_{10}$ and (AGG) ${ }_{10}$ appeared to be colocalized and were primarily distributed in pericentromeric regions on 13-16 chromosomes. $(\mathrm{CAC})_{10}$ and $(\mathrm{CAG})_{10}$ had similar distributions to those observed in H. bogdanii. Signals of (CAC) ${ }_{10}$ and $(\mathrm{CAG})_{10}$ were detectable in 19-22 chromosomes and were stronger on eight or nine chromosomes. The FISH pattern of $(\mathrm{AAT})_{10}$ in $H$. brevisubulatum was distinctly different from that in $H$. bogdanii. (AAT) ${ }_{10}$ produced a stronger hybridization signal in $H$. brevisubulatum than in $H$. bogdanii, primarily in subtelomeric regions on nearly all chromosomes. Signals of (ACG) ${ }_{10}$ were detectable in 13 chromosomes. As with (AAT) ${ }_{10}$, stronger hybridization signals of (GCC) ${ }_{10}$ were detected in $H$. brevisubulatum than in $H$. bogdanii.

$45 \mathrm{~S}$ rDNA produced hybridization signals in subtelomeric regions on seven or eight chromosomes. 5S rDNA was detected in centromeric, pericentromeric, intercalary, or subtelomeric regions on 24 chromosomes. The present karyotypes of $5 \mathrm{~S}$ rDNA and $45 \mathrm{~S}$ rDNA in $H$. brevisubulatum differed from those of $H$. brevisubulatum based on the accessions reported by Taketa et al. (1999). A heterogeneous composition of $5 \mathrm{~S}$ and $45 \mathrm{~S}$ rDNA was observed in a total of five $5 \mathrm{~S} \mathrm{rDNA}$ sites and $1845 \mathrm{~S}$ rDNA sites (Taketa et al. 1999). However, many more $5 \mathrm{~S}$ rDNA sites than $45 \mathrm{~S}$ rDNA sites were detected in our study. This pattern suggests that intraspecific polymorphism may exist among different populations. Distinct pSc119.2 signals were detected in subtelomeric regions on four or five chromosomes.

Furthermore, the technique of genomic in situ hybridization (GISH) was used to determine the polyploidy origin of $H$. brevisubulatum. The result showed that the chromosomes of $H$. brevisubulatum were evenly painted by using labelled genomic DNA of H. bogdanii as the probe (Fig. 5). 


\section{Discussion}

\section{Chromosomal identification and genomic characterization of $\boldsymbol{H}$. bogdanii}

Fifteen of the 18 repetitive sequences produced detectable hybridization in mitotic metaphase chromosomes in $H$. bogdanii. A few repeats, including highly polymorphic sites, can be used to uniquely identify each chromosome. The satellite DNA pAs1 and $5 \mathrm{~S}$ rDNA can be used to distinguish each chromosome in $H$. bogdanii. The SSRs AAC, ACT, CAT, AAG and AGG are also ideal markers for chromosome identification because of their abundance and their large number of polymorphic sites across individual chromosomes (Fig. 6). Thus, individual chromosomes of $H$. bogdanii can be easily identified using the above repeats. In this study, pAs1 repeats were used as a reference marker, and each repetitive sequence was accurately physically mapped.

Most of the repeats produced multiple hybridizations. However, high-intensity hybridizations were always observed on pericentromeric or subtelomeric regions. This implies that the $H$. bogdanii genome contains more repetitive sequences in subtelomeric and pericentromeric parts of the chromosome than in interstitial regions. The distributions of AAC, ACT, and CAT were revealed to be colocalized and were identical in intensity in this study, suggesting that AAC, ACT, and CAT may be evenly distributed in an intermingled way. The same was true for AAG and AGG. Although CAC and CAG were found to be colocalized, different hybridization intensities suggest that their distribution was close rather than intermingled.

Differences between the I and $\mathrm{H}$ genomes can be observed by comparing their distribution of their repetitive sequences. A distinct difference can be observed in a few trinucleotide SSR motifs. In $H$. vulgare (H genome), the AAC, AAG, and AGG motifs are colocalized around the centromere in all chromosomes; ACT produces multiple signals in six chromosome pairs; and CAT produces a strong signal and a weak signal in $4 \mathrm{H}$ and $5 \mathrm{H}$, respectively (Cuadrado and Jouve 2007a) (Fig. 6). Another distinct difference is exhibited by the dinucleotide AG, which was intensely detected around the centromere in H. vulgare (Hagras et al. 2005, Carmona et al. 2013a) but was absent in $H$. bogdanii. Additional differences between $H$. bogdanii and $H$. vulgare can be seen in the distribution patterns of AAT, GCC, CAG, $45 \mathrm{~S}$ rDNA, $5 \mathrm{~S}$ rDNA and $\mathrm{pSc1}$ 19.2. Physical mapping of a few sequences in $H$. marinum (Xa genome) showed that AAC and AAG produced intense and rich patterns of multiple SSR signals that were particularly concentrated in the pericentromeric region and that ACT and CAT were weakly distributed (Carmona et al. 2013b). The pSc119.2 repeat produced subtelomeric signals on nearly all chromosomes in H. marinum (Taketa et al. 2000); however, fewer of these signals were present in $H$. bogdanii. Karyotype analysis of diploid H. murinum $(\mathrm{Xu})$ showed that $A G$ produced intense signals around the centromere in four chromosome pairs and that AAG produced more rich and intense signals than AAC, ACT, and CAT in pericentromeric regions (Cuadrado et al. 2013, Carmona et al. 2013b). In addition, no pSc119.2 hybridization signals were detected in diploid $H$. murinum (Taketa et al. 2000). 


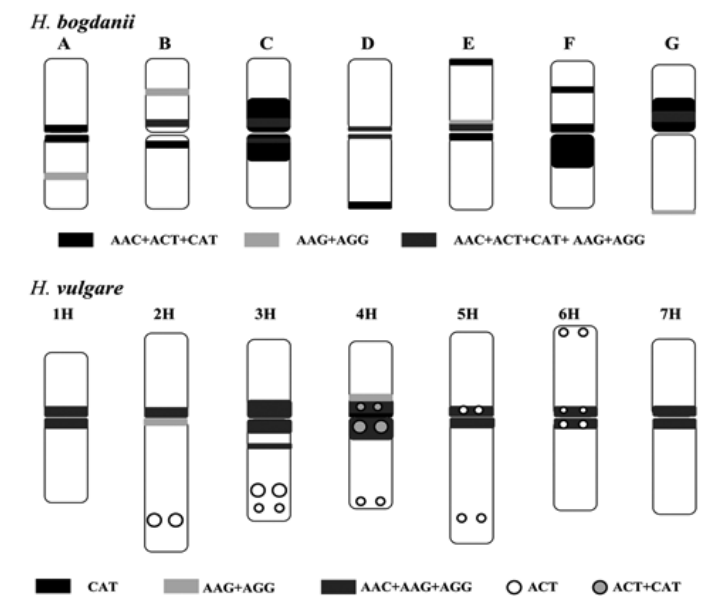

Figure 6. Idiogram of chromosomes of $H$. bogdanii (I genome) and $H$. vulgare (H genome) showing the distributions of the SSRs AAC, ACT, CAT, AAG and AGG. Chromosomal information of H. vulgare is taken from Cuadrado et al. (2007a)

Thus, the genomic composition of the I genome in $H$. bogdanii revealed by the distribution of several repeats was highly different from that of the $\mathrm{H}, \mathrm{Xa}$, and $\mathrm{Xu}$ genomes in Hordeum.

\section{Autopolyploid origin of $\mathrm{H}$. brevisubulatum}

The Hordeum brevisubulatum species complex has a range of cytotypes and can occur in diploid, tetraploid, and hexaploid forms (Bothmer 1979, Dewey 1979, Linde-Laursen et al. 1980). Earlier studies have shown that polyploids in the H. brevisubulatum complex may be autoploids (Bothmer 1979, Dewey 1979). Data from meiotic pairing in the hybrids indicate that autoploidy characterizes the entire complex, with one "basic" genome (Landström et al. 1984). The karyotype of tetraploid $H$. brevisubulatum was not shown to be a strictly doubled karyotype from a diploid form. However, similar chromosomal distributions of many repetitive sequences between $H$. bogdanii and $H$. brevisubulatum were revealed in this study. Specifically, colocalized distributions of AAC, ACT, and CAT in pericentromeric regions in nearly all chromosomes and colocalized distributions of AAG and AGG in most chromosomes were observed in $H$. brevisubulatum. Nearly double the number of $45 \mathrm{~S} \mathrm{rDNA}$ and $5 \mathrm{~S} \mathrm{rDNA}$ sites in $H$. bogdanii were also detected in $H$. brevisubulatum. In addition, no genomic differentiation was detected in $H$. brevisubulatum by GISH with labelled genomic DNA of $H$. bogdanii. This pattern strongly suggests an autopolyploid origin of $H$. brevisubulatum from an I genome.

Several examples of structural rearrangements of chromosomes at the population level in the H. brevisubulatum complex have been reported (Landström et al. 1984). In 
this study, high levels of chromosomal variation, including variation in chromosome number and chromosome structure, were observed in the population under investigation. This observation implies that there is a high degree of instability of the autoploid genome in $H$. brevisubulatum. Karyotype variations have recently been reported in resynthesized and naturally formed allopolyploid species and in natural hybrids produced by polyploid homoploid hybridization (Chester et al. 2012, Lipman et al. 2013). Aneuploidy, inter- and intragenomic rearrangements, and the loss of repeats were frequently detected in early generations. As a natural species, $H$. brevisubulatum should be the product of a long evolutionary history. Hordeum brevisubulatum has been considered to be self-incompatible (Bothmer 1979). Although autopolyploidy may result from genome doubling within a single individual, most natural autopolyploids likely formed via some degree of hybridization, involving, for example, individuals from genetically differentiated populations (Stebbins 1985, Soltis and Soltis 1989). Thus, more studies are needed to determine whether karyotype variation in $H$. brevisubulatum might be affected by hybridization between genetically differentiated biotypes.

Major genetic changes, including the loss of homologs and DNA sequences, have been documented in recently formed polyploid species (Soltis et al. 2004, Abbott and Lowe 2004, Matyášek et al. 2007). Compared with the composition of the I genome in $H$. bogdanii, the distributions of a few repetitive sequences in $H$. brevisubulatum might be strongly altered by increases or decreases in copy number. The trinucleotide SSR motifs AAC, ACT, and CAT are primarily located in pericentromeric regions in all the chromosomes in $H$. bogdanii, but they are absent in a few chromosomes in $H$. brevisubulatum, possibly because of decreases in copy number. The distributions of AAG and AGG are similar. In contrast, the higher abundance of ATT and GCC in H. brevisulatum indicates that there is an increase in copy number that accompanies polyploidization.

\section{Evolutionary trends of repetitive DNA sequences during genome differentiation}

Repetitive DNA sequences are the main components of heterochromatin and are subject to rapid change. Such changes in the distribution of repetitive DNA sequences are one of the driving forces of genome evolution and speciation. One proposed function of repetitive sequences may be related to higher-order molecular structure (Redi et al. 2001). However, the molecular mechanisms by which genomes change are unknown (Plohl et al. 2002).

The SSR motif AAC was shown to be distributed mainly in the pericentromeric regions of the $\mathrm{I}, \mathrm{H}, \mathrm{Xa}$, and $\mathrm{Xu}$ genomes. In addition, the wide distribution of $\mathrm{AAC}$ has been detected in wheat and the genus Secale (Cuadrado et al. 2000 Cuadrado and Jouve 2002). This wide distribution suggests that AAC repeats have an ancient origin in Triticeae species. The distribution of AAC in the I genome was revealed to be evenly co-localized with ACT and CAT. However, the distributions of AAC, ACT, and CAT are largely differentiated in the $\mathrm{H}, \mathrm{Xa}$, and Xu genomes. Variation in the distribution of repetitive sequences may be related to the amplification and deletion of repeat copies. 
AAC, ACT, and CAT motifs in the I genome may have been co-amplified or deleted as a single repetitive unit. However, AAC, ACT, and CAT in other genomes have evolved independently. AAG exhibits the most chromosomal variation both within and between taxa (Pedersen et al. 1996, Pedersen and Langridge 1997, Cuadrado et al. 2008, Carmona et al. 2013a). AAG has been suggested to be more predisposed to being amplified or deleted relative to other repetitive sequences as a consequence of independent events in different lineages (Carmona et al. 2013b). The fact that AAG is evenly colocalized with AGG in the I genome suggests that the same evolutionary mechanism also drives variation in the chromosomal distribution of AAC, ACT, and CAT.

$\mathrm{AC}$ repeat sites are remarkably similar and have been shown to exhibit uniformly dispersed hybridization along the euchromatic portion of metaphase chromosomes in humans and barley and in the metaphase and polytene chromosomes of Drosophila melanogaster (Meigen, 1830) (Cuadrado and Jouve 2007b). In this study, AC repeat sites had a dispersed distribution. High-intensity hybridization bands for AC repeats were observed in subtelomeric and pericentromeric regions on a few chromosomes in the I genome. This atypical distribution suggests that there is a more complicated organization or function of AC repeats in plant genomes.

The sequence pAs1 contains Afa family sequences. The Afa family sequence was abundant in the four basic genomes of the genus Hordeum, and the hybridization patterns differed among the diploid species (Taketa et al. 2000). The distribution of the Afa family is more dispersed than those of the other tandem repetitive sequences. This dispersed distribution implies that the Afa family may be more commonly distributed in gene rich regions. In our study, despite the autopolyploidy origin of $H$. brevisubulatum from an I genome species, the distribution of pAs1 sequences in $H$. brevisubulatum was highly variable relative to those in diploid $H$. bogdanii. The Afa family of sequences may play an important role in the differentiation of either genome or species in the genus Hordeum.

Variation in the abundance and distribution of repetitive sequences and the shared distribution of the same repeats between different genomes suggest that repetitive sequences play a key role in both the structure and function of the genomes of higher eukaryotes (Cuadrado et al. 2008). To date, most information on the distribution of repeats in Hordeum genomes comes from physical mapping analysis of mitotic metaphase chromosomes, which are highly packaged. Physical mapping of repetitive sequences on extended chromosomes, such as during the pachytene stage of meiosis, may provide more valuable information on the chromosomal distribution of repeats and may help elucidate the evolution and genomic function of repetitive sequences.

\section{Conclusion}

Fifteen repetitive sequences, including SSR motif $\mathrm{AC}$ and all possible trinucleotide motifs and satellite DNAs pAs1, 5S rDNA, 45s rDNA, and pSc119.2, were accurately physically mapped on individual chromosomes in the I genome in H. bogdanii. High 
genome instability was revealed in tetraploid $H$. brevisubulatum. The similar distribution of the repeats in both species suggests an autopolyploid origin of $H$. brevisubulatum from an I genome species. Comparative cytogenetic analysis between the I genome and other genomes in Hordeum showed that the distribution of a few repeats differed. Colocalization of motifs AAC, ACT, and CAT and colocalization of motifs $\mathrm{AAG}$ and $\mathrm{AGG}$ is characteristic of the I genome.

\section{Acknowledgements}

This work was supported by the Natural Science Foundation of Qinghai Province (no. 2015-ZJ-903).

\section{References}

Abbott RJ, Lowe AJ (2004) Origins, establishment and evolution of new polyploid species: Senecio cambrensis and S. eboracensis in the British Isles. Biological Journal of the Linnean Society 82: 467-74. doi: 10.1111/j.1095-8312.2004.00333.x

Badaeva ED, Amosova AV, Muravenko OV, Samatadze TE, Chikida NN, Zelenin AV, Gill BS (2002) Genome differentiation in Aegilops. 3. Evolution of the D-genome cluster. Plant Systematics and Evolution 231(1-4): 163-190. doi: 10.1007/s006060200018

Badaeva ED, Friebe B, Gill BS (1996) Genome differentiation in Aegilops. 1. Distribution of highly repetitive DNA sequences on chromosomes of diploid species. Genome 39(2): 293-306. doi: 10.1139/g96-040

Bedbrook JR, Jones J, O'Dell M, Thompson RD, Flavell RB (1980) A molecular description of telomeric heterochromatin in Secale species. Cell 19: 545-560. doi: 10.1016/00928674(80)90529-2

Blattner FR (2009) Progress in phylogenetic analysis and a new infrageneric classification of the barley genus Hordeum (Poaceae: Triticeae). Breeding Science 59(5): 471-480. doi: 10.1270 /jsbbs. 59.471

Bothmer R (1979) Revision of the Asiatic taxa of Hordeum sect. Stenostachys. Botanisk Tidsskrift 74: 117-146.

Bothmer R, Jacobsen N, Baden C, Jorgensen RB, Linde-Laursen I (1995) An ecogeographical study of the genus Hordeum, 2nd edn. Systematic and ecogeographical studies on crop Gene pools 7. IBPGR, Rome.

Bothmer R, Flink J, Landström T (1986) Meiosis in interspecific Hordeum hybrids. I. Diploid combinations. Canadian Journal of Genetics and Cytology 28(4): 525-535. doi: 10.1139/ g86-077

Carmona A, Friero E, de Bustos A, Jouve N, Cuadrado A (2013a) Cytogenetic diversity of SSR motifs within and between Hordeum species carrying the $\mathrm{H}$ genome: $H$. vulgare $\mathrm{L}$. and H. bulbosum L. Theoretical and Applied Genetics 126(4): 949-961. doi: 10.1007/ s00122-012-2028-y 
Carmona A, Friero E, de Bustos A, Jouve N, Cuadrado A (2013b) The evolutionary history of sea barley (Hordeum marinum) revealed by comparative physical mapping of repetitive DNA. Annals of Botany 112(9): 1845-1855. doi: 10.1093/aob/mct245

Chester M, Gallagher JP, Symonds VV, Cruz da Silva AV, Mavrodiev EV, Leitch AR, et al. (2012) Extensive chromosomal variation in a recently formed natural allopolyploid species, Tragopogon miscellus (Asteraceae). Proceeding of the National Academy of Sciences of the United States of America 109(4): 1176-1181. doi: 10.1073/pnas.1112041109

Cuadrado A, Cardoso M, Jouve N (2008) Physical organisation of simple sequence repeats (SSRs) in Triticeae: structural, functional and evolutionary implications. Cytogenetic and Genome Research 120(3-4): 210-219. doi: 10.1159/000121069

Cuadrado A, Carmona A, Jouve N (2013) Chromosomal Characterization of the Three Subgenomes in the Polyploids of Hordeum murinum L.: New Insight into the Evolution of This Complex. PLoS ONE 8(12): e81385. doi: 10.1371/journal.pone.0081385

Cuadrado A, Ceoloni C, Jouve N (1995) Variation in highly repetitive DNA composition of heterochromatin in rye studied by fluorescence in situ hybridization. Genome 38: 101-1069. doi: $10.1139 / \mathrm{g} 95-142$

Cuadrado A, Jouve N (2002) Evolutionary trends of different repetitive DNA sequences during speciation in the genus Secale. Journal of Heredity 93(5): 339-345. doi: 10.1093/ jhered/93.5.339

Cuadrado A, Jouve N (2007a) The nonrandom distribution of long clusters of all possible classes of trinucleotide repeats in barley chromosomes. Chromosome Research 15(6): 711-720. doi: 10.1007/s10577-007-1156-8

Cuadrado A, Jouve N (2007b) Similarities in the chromosomal distribution of AG and AC repeats within and between Drosophila, human and barley chromosomes. Cytogenetic and Genome Research 119(1-2): 91-99. doi: 10.1159/000109624

Danilova TV, Friebe B, Gill BS (2012) Single-copy gene fluorescence in situ hybridization and genome analysis: Acc-2 loci mark evolutionary chromosomal rearrangements in wheat. Chromosoma 121(6): 597-611. doi: 10.1007/s00412-012-0384-7

Dewey DR (1979) The Hordeum vioceum complex of Iran. American Journal of Botany 66: 166-172. doi: 10.2307/2442520

Dou QW, Chen ZG, Liu YA, Tsujimoto H (2009) High frequency of karyotype variation revealed by sequential FISH and GISH in plateau perennial grass forage Elymus nutans. Breeding science 59(5): 651-656. doi: 10.1270/jsbbs.59.651

Flavell RB, Bennett MD, Smith JB, Smith DB (1974) Genome size and the proportion of repeated nucleotide sequence DNA in plants. Biochemical Genetics 12(4): 257-269. doi: 10.1007/BF00485947

Flavell RB, Rimpau J, Smith DB (1977) Repeated sequence DNA relationships in four cereal genomes. Chromosoma 63: 205-222. doi: 10.1007/BF00327450

Fukui K, Kamisugi Y, Sakai F (1994) Physical mapping of 5 S rDNA loci by direct-cloned biotinylated probes in barley chromosomes. Genome 37(1): 105-111. doi: 10.1139/g94-013

Hagras A, Kishii M, Tanaka H, Sato K, Tsujimoto H (2005) Genomic differentiation of Hordeum chilense from $H$. vulgare as revealed by repetitive and EST sequences. Genes \& Genetic Systems 80(3): 147-159. doi: 10.1266/ggs.80.147 
Heslop-Harrison J, Schwarzacher T (2011) Organisation of the plant genome in chromosomes.

The Plant Journal 66(1): 18-33. doi: 10.1111/j.1365-313X.2011.04544.x

Jiang J, Gill BS (2006) Current status and the future of fluorescence in situ hybridization (FISH) in plant genome research. Genome 49(9): 1057-1068. doi: 10.1139/G06-076

Kato A (1999) Air drying method using nitrous oxide for chromosome counting in maize.

Biotechnic \& Histochemistry 74(3): 160-166. doi: 10.3109/10520299909047968

Landström T, Bothmer R, Dewey DR (1984) Genomic relationships in the Hordeum brevisubulatum complex. Canadian Journal of Genetics and Cytology 26(5): 569-577. doi: 10.1139/g84-090

Linde-Laursen I, Bothmer R, Jacobsen N (1980) Giemsa C-banding in Asiatic taxa of Hordeum section Stenostachys with notes on chromosome morphology. Hereditas 93(2): 235-254. doi: 10.1111/j.1601-5223.1980.tb01364.x

Linde-Laursen I, Bothmer R, Jacobsen N (1992) Relationships in the genus Hordeum: Giemsa C-banded karyotypes. Hereditas 116(1-2): 111-116. doi: 10.1111/j.1601-5223.1992. tb00213.x

Lipman MJ, Chester M, Soltis PS, Soltis DE (2013) Natural hybrids between Tragopogon mirus and T. miscellus (Asteraceae): a new perspective on karyotypic changes following hybridization at the polyploid level. American Journal of Botany 100(10): 2016-2022. doi: 10.3732/ajb.1300036

Matyášek R, Tate JA, Lim YK, Šrubařová H, Koh J, Leitch AR, Kovařík A (2007) Concerted evolution of rDNA in recently formed Tragopogon allotetraploids is typically associated with an inverse correlation between gene copy number and expression. Genetics 176(4): 2509-2519. doi: 10.1534/genetics.107.072751

Pedersen C, Rasmussen SK, Linde-Laursen I (1996) Genome and chromosome identification in cultivated barley and related species of the Triticeae (Poaceae) by in situ hybridization with the GAA-satellite sequence. Genome 39(1): 93-104. doi: 10.1139/g96-013

Pedersen C, Langridge P (1997) Identification of the entire chromosome complement of bread wheat by two-colour FISH. Genome 40(5): 589-593. doi: 10.1139/g97-077

Plohl M, Luchetti A, Mestrovic N, Mantovani B (2002) Satellite DNAs between selfishness and functionality: structure, genomics and evolution of tandem repeats in centromeric (hetero) chromatin. Gene 409: 72-82. doi: 10.1016/j.gene.2007.11.013

Rayburn AL, Gill BS (1986) Isolation of a D-genome specific repeated DNA sequence from Aegilops squarrosa. Plant Molecular Biology Reporter 4: 102-109. doi: 10.1007/BF02732107

Redi C, Garagna S, Zacharias H, Zuccotti M, Capanna E (2001) The other chromatin. Chromosoma 110(3): 136-147. doi: 10.1007/s004120000114

Soltis DE, Soltis PS (1989) Genetic consequences of autopolyploidy in Tolmiea (Saxifragaceae). Evolution 43: 586-94. doi: 10.2307/2409061

Soltis DE, Soltis PS, Pires JC, Kovarik A, Tate J, Mavrodiev E (2004) Recent and recurrent polyploidy in Tragopogon (Asteraceae): cytogenetic, genomic and genetic comparisons. Biological Journal of the Linnean Society 82: 485-501. doi: 10.1111/j.1095-8312.2004.00335.x Stebbins GL (1985) Polyploidy, hybridization, and the invasion of new habitats. Annals of the Missouri Botanical Garden 72: 824-32. doi: 10.2307/2399224 
Taketa S, Ando H, Takeda K, Harrison G, Heslop-Harrison J (2000) The distribution, organization and evolution of two abundant and widespread repetitive DNA sequences in the genus Hordeum. Theoretical and Applied Genetics 100(2): 169-176. doi: 10.1007/ s001220050023

Taketa S, Ando H, Takeda K, Bothmer R (2001) Physical locations of 5S and 18S-25S rDNA in Asian and American diploid Hordeum species with the I genome. Heredity 86(5): 522530. doi: 10.1046/j.1365-2540.2001.00768.x

Taketa S, Harrison G, Heslop-Harrison J (1999) Comparative physical mapping of the 5 S and 18S-25S rDNA in nine wild Hordeum species and cytotypes. Theoretical and Applied Genetics 98(1): 1-9. doi: 10.1007/s001220051033

Tang Z, Yang Z, Fu S (2014) Oligonucleotides replacing the roles of repetitive sequences pAs1, pSc119. 2, pTa-535, pTa71, CCS1, and pAWRC. 1 for FISH analysis. Journal of Applied Genetics 55(3): 313-318. doi: 10.1007/s13353-014-0215-z

Tsujimoto H, Mukai Y, Akagawa K, Nagaki K, Fujigaki J, Yamamoto M, Sasakuma T (1997) Identification of individual barley chromosomes based on repetitive sequences: conservative distribution of Afa-family repetitive sequences on the chromosomes of barley and wheat. Genes \& Genetic Systems 72(5): 303-309. doi: 10.1266/ggs.72.303

Yang J, Yen C, Lu B, Bothmer R (1987) The genus Hordeum (Gramineae) in China. Variation and distribution. Willdenowia 16: 479-490. 